\title{
In vitro evaluation of artificial joints: a comprehensive review
}

\author{
Gang Shen $^{1} \cdot$ Ju-Fan Zhang ${ }^{1} \cdot$ Feng-Zhou Fang ${ }^{1,2}$
}

Received: 12 September 2018/ Accepted: 17 December 2018/Published online: 21 February 2019

(C) The Author(s) 2019

\begin{abstract}
Development of artificial joints is a great progress for joint replacement operations of human being, but the short longevity of prostheses has concerned both industries and researchers since the advent of modern implants. Thus, continuous improvements have been made alongside the clinical applications. The methodologies to inspect the potential properties of new designs have also seen many advances. The test machines have evolved from easy pin-on-disk configuration to the modern joint simulator. Besides, various wear traces provided by testing machines are investigated greatly, and multi-directional files are recommended as motion profiles for in vitro testing. The typical testing parameters, like sliding speed or loading profile, are discussed in this article, and their working mechanisms are described in detail. Meanwhile, the calf or bovine serum has been regarded as the gold standard for testing lubricant, and the future trend of fluid will be focused on investigating the effects of lubricant composites on the tribological properties. Hard particles and proteins are discussed as well, and their effects on the wear mechanisms are the focus. Finally, various approaches to measure the wear rate, wear factor and the thickness of lubricant film are presented, and suggestions are given for the development in future.
\end{abstract}

Feng-Zhou Fang

fengzhou.fang@ucd.ie

1 Centre of Micro/Nano Manufacturing Technology (MNMTDublin), University College Dublin, Dublin, Ireland

2 State Key Laboratory of Precision Measuring Technology and Instruments, Centre of Micro/Nano Manufacturing Technology (MNMT), Tianjin University, Tianjin 300072, China
Keywords Artificial joint - Methodology · Testing parameter . Wear rate

\section{Introduction}

Total joint replacement (TJR) is regarded as one of the most successful surgery operations in bio-medical area. It is generally recognised that an increasing number of people would take the joint replacement operations in the future due to an aging population and the demand for a more active lifestyle [1, 2]. Kurtz et al. [3] predicted that the primary hip and knee arthroplasty would grow by $174 \%$ and $673 \%$, respectively, in the United States between 2005 and 2030. Besides, more and more young people need the implanted prostheses with a longer lifespan and higher stability [4]. However, the short lifespan of the commercial prostheses, approximately 15 years [5], limits their applications for the young patients. It is widely believed that the reactions between wear particles and natural bones are the main failure mechanisms. Thus, prostheses with new designs and novel manufacturing methods need to be introduced to replace the current products [6,7], aiming at improving the wear properties. Several modifications of the polyethylene (PE)-based joints, like crosslinking or reinforcing PE [8], adopting different counter-bearings [9] and changing the chemical properties of bearing surfaces [10], have been proposed by many researchers in recent years. The introduction of these modifications results in the need for proper evaluation procedures to identify the promising designs for future bioimplants.

Due to the complex environment of the human body, it is almost impossible to exactly replicate the surrounding lubricants, loading profiles and the motion tracks experienced by the implanted joints during the in vitro testing. 
The International Organization for Standardization (ISO) provided researchers with several rules, based on which the in vitro evaluation of the newly-designed artificial joints would be performed before clinical applications. However, it has been reported that the standard only offers the loading profiles and motion tracks under the normal walking conditions [11, 12]. Bowsher and Shelton [13] once investigated the differences of the peak loads among walking, jogging, and stumbling, and found that the peak load during stumbling was two times more than that under the normal walking condition. Hard particles are also proved to cause deteriorations to the bearing surfaces of implanted joints [14], which has not been included in most laboratory tests. The difficulties of getting enough natural synovial fluids (SF) push researchers into using simulated lubricants. Although calf or bovine serum is believed to provide a proper testing environment, there is an evidence that a better synthetic lubricant is needed for the performance evaluation [15]. The main discrepancy between SF and simulated lubricants is the various protein contents and concentrations, and this has attracted many researchers in investigating the functionality of proteins on the tribological properties [16]. Here, the role of the protein layer on the wear resistance of bio-implants is comprehensively reviewed. Generally, the tribological analysis of artificial joints is divided into two categories: wear mechanism and lubricant regime. The wear properties are usually represented as wear rate or wear factor, and the investigation of lubricant regime relies on the measurement of film thickness.

As a result, the testing environments vary a lot among different researchers. Fisher et al. [17] reported the differences of wear factors among various research groups and concluded that this phenomenon was due to the factor that different test conditions, like sliding velocity and lubricant, were adopted by different scholars. Besides, it was concluded by many researchers that the wear rates produced under the current testing parameters were lower than clinical data $[18,19]$, which was partly attributed to the improper replication of the in vivo situation. This review covers a detailed discussion of the testing principles. A thorough review of previous testing parameters was also conducted, along with their working mechanisms. Finally, a description of the measuring method is included. This paper would benefit researchers or engineers who are engaged in designing and testing artificial joints.

\section{Testing approaches}

A laboratory test is an essential procedure before the clinical application of newly-designed artificial joints. The first tribological testing simulator was set up in the 1960s for evaluating Charnley's hip prostheses [20]. Afterward, large quantities of testing machines were set up, and the aim of those newly-built simulators was to mimic a more proper in vivo environment [21]. Wear mechanisms would be the major criterion for checking the validity of the in vitro testing data. Thus, wear mechanisms from the laboratory testing are always compared to those from the clinical situation. Besides, the motion tracks provided by the testing machine are important factors affecting the wear mechanisms and have evolved from the linearly unidirectional profiles to the multidirectional situation.

Nowadays, the full pre-clinical evaluations of artificial joints are conducted in two steps. The first step is to use the pin/ball-on-disk (POD) configuration, which is regarded as a quick and easy way to do the simulation testing. The second step would utilize the sophisticated hip or knee simulator, which could offer more precise data compared to that from POD. In this paper, the working mechanisms of both simulators are described in detail.

\subsection{Pin/ball-on-disk}

The application of pin/ball-on-disk machine in the field of orthopedic evaluation can date back to the 1960s, which was used to check the wear rate of UHMWPE-based joints [22]. It is widely recognised that POD configuration is designed to perform the fast evaluation of the artificial joints, and the testing results could be seen as a prediction for the in vivo performance.

The configurations could be divided into two categories: pin-on-disk (see Fig. 1 [23]) and ball-on-disk (see Fig. 2 23), which could both be used for testing artificial hip or knee joints. In general, the ball-on-disk configuration is more suitable for testing artificial knee joints, since this configuration bears more resemblance to the anatomical figure of natural knee joints. The theoretical basis of using the POD simulator machine could be described by the Archard's law [24], which assumed that the wear of joint material only depended on the normal load and the total sliding distance. Based on this theory, unidirectional POD simulators attracted researchers' interests in the last

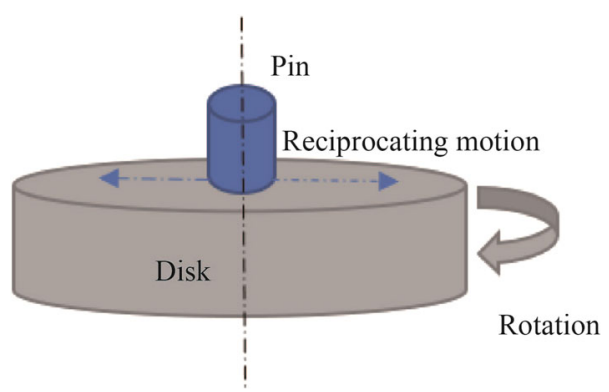

Fig. 1 Pin-on-disk machine [23] 


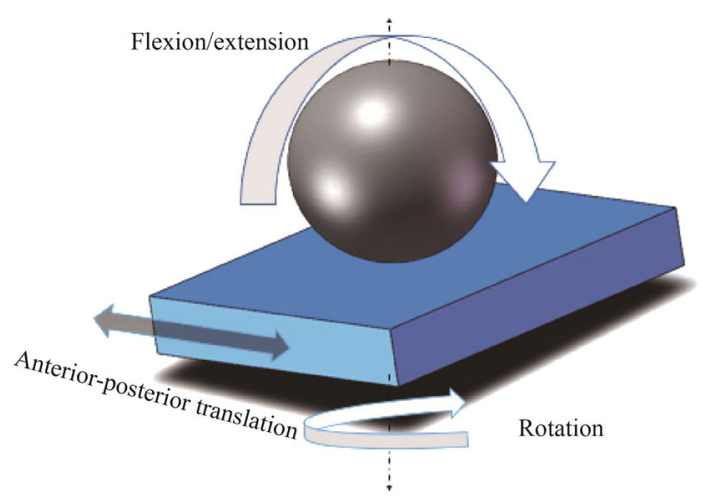

Fig. 2 Ball-on-disk machine [23]

century, and this machine could only provide the reciprocation of the pin or the rotating motion of the disk (see Fig. 1). The unidirectional testing machine was first used to evaluate the wear performance of PTFE-based hip implants in the early 1960s [20], and this kind of testing machine achieved a huge success in the first three decades after the initial set up. However, it was reported that unidirectional profiles could not replicate the real in vivo situation of the polymer-based prostheses [25]. Wang et al. [26] concluded that the wear rates based on the unidirectional testing machine were at least two orders of magnitude smaller than clinical data. Also, it was reported that wear mechanisms from the unidirectional POD simulator were different from the clinical situation [27]. Wang [25] performed a comprehensive investigation on the effects of wear tracks onto the testing accuracy and found the importance of cross shear for the accuracy of laboratory testing results.

Following the unidirectional debacle, multidirectional POD simulator has attracted much attentions in the last 20 years. Compared to the unidirectional testing machine, multidirectional POD simulator could provide a more complex motion profile and has been regarded by many researchers as a proper in vitro testing apparatus [21, 28]. Bragdon et al. [29] compared the testing results from unidirectional motion to those from rectangular motion and found that the wear rates or the surface morphologies of the multidirectional POD simulation were more consistent with that of the explanted prostheses. Besides, they gave a brief explanation on the differences of testing data: the cross-shear forces provided by the multidirectional motion were the predominant factors in the removal of polyethylene material. Some other researchers [30] also came to a similar conclusion that material along the main sliding path was straining-hardened, due to which the wear resistance was improved. In addition, it was widely recognised that the wear traces on the surface of implanted joints were near an elliptical or rectangular shape for most patients, although some patients exhibited more square-like wear tracks [31]. As a result, the wear mechanisms of polymer- based prostheses under multi directional conditions are more similar to the clinical situation, and lots of efforts have been paid to investigate the functionality of the wear path. Wang [25] once did a theoretical analysis on the influence of multidirectional motion on the wear performance of UHMWPE and found that the materials along the sliding direction would be strengthened, but the wear resistance in the perpendicular direction would be weakened. Thus, the aspect ratio ( $A / B$, see Fig. 3 [30]) would be an important factor for the in vitro wear testing, and the equation describing the correlation between wear factor and aspect ratio has been established [31]

$k \propto \frac{A}{A+B}$,

where $k$ means the wear factor; $A$ and $B$ are the width and length of the rectangular tracks, respectively. Wang [25] proposed that the maximum wear loss of UHMWPE would be in the condition when $A=B$ or $A /(A+B)=0.5$. However, this theoretical prediction could not be proved by the laboratory tests. Turell et al. [30, 31] compared the testing results of six sets of aspect ratio and found that the maximum wear loss would occur when the aspect ratio was 2.33. Other aspect ratios, contributing to the maximum polymer wear loss, have also been reported by some researchers [32, 33]. Similarly, the elliptical wear track, ranging from a circle to a line, was investigated to check its effect on the wear performance of UHMWPE [34]. Generally, the value of aspect ratio has an important effect on the in vitro testing results of UHMWPE-based implants, and this parameter should be considered when comparing the testing data from different groups. However, Korduba and Wang [35] found that the wear rate of highly-crosslinked polyethylene (HXPE) was almost the same under the condition of unidirectional and multidirectional wear tracks. This was due to the crosslinks within HXPE hindering the orientation softening. However, the effects of wear trace on other material combinations, like metal-onmetal and ceramic-on-ceramic, have not been investigated. Thus, future works should pay attention to the functionality of cross shear on hard-on-hard configuration.

To sum up, the main purpose of POD simulator testing is to give comparative wear rates between different designs or material combinations instead of absolutely precise testing results [21]. Also, it is widely recognised that

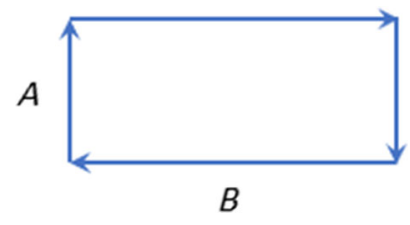

Fig. 3 Rectangular wear motion of artificial joints [30] 
optimal testing parameters for pin/ball-on-disk configuration to closely imitate the in vivo wear conditions may not be available. Besides, the value of aspect ratio for pin-ondisk simulation would affect the wearing mechanisms, and thus, the testing results. It could be said that POD evaluation provided a fast but not optimal testing procedure, and the further test should be done using the modern hip or knee simulator, which could give more proper testing conditions, before the clinical application.

\subsection{Hip and knee simulators}

Unlike POD configuration, full implant simulators can provide a more elaborated testing environment by considering most factors: implant geometry, materials, lubricant, loading and motion profiles. Also, the anatomical position of the two components during the evaluation process could mimic the real in vivo condition. Besides, there is a standard (ISO 14242) for us to refer to when using the physiological simulators. In addition, there is a general rule for the orthopedic industry that any newly-designed products must be evaluated using the full joint simulator for at least 5 million cycles before clinical application. In recent years, the sophisticated machines have been adopted by many researchers to perform thorough tests for bioimplants instead of the pin-on-disk machine. As we can see from Table 1, the full joint simulator could be used to test almost all the aspects of artificial joints: ranging from evaluating the wear performance of most conventional material combination to the research on finding bio-mimical wear tracks [19, 36-43]. Meanwhile, there were more research works using the hip simulator than the full knee simulator, which might be due to the historical fact that total hip arthroplasty was more frequently carried out in the last century. However, this trend has changed over the last decade, and there would be more people needing to take the knee implant operations in the future [3], which would result in more research works using the physiological knee simulators.

Generally, the pin-on-disk machine is the main testing apparatus for evaluating the newly-designed artificial joints. Meanwhile, it is compulsory to test the bioimplants using the full hip or knee joint simulator before the clinical application. The common problem for both testing machines is to find the proper simulating environment. For physiological joint simulator, researchers may need to consider more about the implement of severe wear, while wear trace, sliding velocity, and loading profile are more important factors for the laboratory evaluation based on the POD simulator.

\section{Influencing factors}

The testing parameters are crucial to the accuracy of the tribological simulation. Due to the complex environment of the human body, it is hard to precisely replicate the in vivo situation. Besides, the simplified structure of POD machines would stop them from providing an optimal testing environment for the artificial joints. Many factors, like the precipitation of hard particles and the lack of in vivo loading profiles, would also result in the impossibility of exhaustively evaluating artificial joints based on the full joints simulator. As a result, most researches were conducted under a testing situation, which could, to some extent, mimic the clinical walking condition.

The main development, which has happened in the laboratory testing in recent years, is that the increasing number of tests has been performed under severe wear conditions $[44,45]$. Since there is not any general rule offering the guidance on conducting these laboratory experiments, the testing conditions vary a lot among different research groups. In this chapter, sliding velocity, contact pressure, hard particles, and lubricant would be reviewed, and some conclusions would also be made to

Table 1 Research on physiological simulator for the in vitro evaluation of artificial joints

\begin{tabular}{lll}
\hline Author & Implant category & Research purpose \\
\hline Firkins et al. [36] & Hip & Wear mechanisms of metal-on-metal hip implants \\
Bigsby et al. [19] & Hip & Wear rate of UHMWPE \\
Tipper et al. [37] & Hip & Performance evaluation of various material combinations \\
Ali et al. [38] & Hip & Effect of simulator design on the wear mechanisms of metal-on-polymer bioimplants \\
Moro et al. [39] & Hip & Influence of phospholipid polymer coating on the wear performance of artificial joints \\
Herrmann et al. [40] & Hip & Stability of artificial hip joints, with focus on the dislocation \\
Kawanabe et al. [41] & Knee & Effect of wear tracks on the wear performance of UHMWPE \\
Dowson et al. [42] & Knee & Penetration of metal femoral part into the polymer tibial plate \\
Tipper et al. [43] & Hip and knee & Size and morphology of UHMWPE wear debris \\
\hline
\end{tabular}


provide some instructions for conducting tribological testing, including the normal walking conditions and severe wear testing environment. Other parameters, like ambient temperature, surface topography, sliding distance and so on, would also be covered in this chapter.

\subsection{Sliding velocity}

The tribological evaluations of artificial joints have been conducted based on a model, which aims at replicating the environment encountered by implanted joints during normal walking, since the advent of modern prostheses in the 1960s [13]. Sliding velocity is an important factor for the in vitro testing results. It was reported by Fisher et al. [17] that the sliding velocity of the implanted artificial hip joints varied a lot among different people, ranging from 0 to $60 \mathrm{~mm} / \mathrm{s}$. Saikko [46] investigated the influence of contact area under the normal walking situation, the speed of which varies between 0 and $31.4 \mathrm{~mm} / \mathrm{s}$. The idea that laboratory tests should be conducted under the normal walking condition, is also favored by many other researchers [47-49]. Besides, relevant ISO standard is also set up based on the clinical data under normal walking conditions.

However, there are some drawbacks of using the velocity range based on normal walking data during the in vitro experiments. Firstly, it would take several months finishing 5 million cycles of motion, which is needed to be done before clinical application, under such low speed. Under this situation, Fisher et al. [17] compared the tribological performance of artificial joints under two distinctive speeds, $35 \mathrm{~mm} / \mathrm{s}$ and $240 \mathrm{~mm} / \mathrm{s}$, and found that the variations of testing speed would affect little on the final data, thus suggesting increasing the sliding velocity during simulation testing. Secondly, the simulated environment, under the velocity of normal walking condition, cannot represent the real motion profiles encountered by implanted prostheses, especially those of the young patients. Bowsher and Shelton [13] reported the occurrence of jogging, a severe exercise with faster motion and heavier loading, at the frequency of 1.75 would significantly increase the simulated wear. In addition, it is widely accepted that some other exercises, like running, jumping or stumbling, have faster sliding speed, sudsequently resulting in the change of wear rate [50]. As a result, sliding velocity is one of the most important factors for the pre-clinical evaluation, and a more complex profile of sliding velocity is needed to conduct the simulation testing [51]. However, the sliding velocity was various among different research groups. As can be seen from Table 2, the speed in the laboratory testing could range from 0 to $2000 \mathrm{~mm} / \mathrm{s}$ [15, 46, 51-54]. Besides, some tests are carried out at a constant speed, while the sliding velocity of other simulations is continually changing. Dressler et al. [55] claimed that the change in the sliding direction, which happened frequently in the clinical situation, could greatly increase the wear rate. As a result, it was widely reported that wear factors and friction coefficient would be distinctively different among various researchers due to the different velocity conditions [56, 57].

In general, sliding velocity would have an effect on the results of the tribological evaluation, and this phenomenon is partly attributed to changes in lubrication regime under different testing speeds. Stribeck curve is a widely used diagram to describe the relationship between the friction coefficient and sliding velocity $[58,59]$. Figure 4 shows a typical Stribeck curve, and it can be easily found that testing speed has a significant influence on the lubricant regime, thus affecting the tribological performance. It is recognised that the increase of sliding velocity would contribute to the formation of thicker lubricant film [60]. In other words, at higher testing speed, it is easier to achieve the hydrodynamic situation, under which the tribological performance of artificial joints is much better than that under boundary lubrication. However, when the film thickness is much larger than the surface roughness, there would be the occurrence of internal friction force between fluid layers, producing additional friction force to the artificial joints [61]. Apart from the effect on the formation of lubricant film, it was also reported that sliding velocity would influence the deposition of proteins on the bearing surfaces, and the protein precipitation could alter the lubricant regime of bioimplants [62, 63]. Besides, Zhang et al. [51] reported that sliding velocity would affect the interfacial temperature, which could affect the rate of protein adsorption [64]. In addition, the changes in temperature could affect the viscosity of the lubricant, thus resulting in the altering of lubricant regimes [65].

Generally, sliding velocity would influence the accuracy of in vitro testing to some extent. Higher velocity would alter the tribological performance of hip and knee prostheses, due to the increased film thickness and protein precipitation. Also, the change in sliding direction would make the wear performance more severe. Thus, a more realistic sliding profile should be adopted to check the functionality of newly-designed artificial joints in modern hip or knee simulators before clinical applications. Here, it is proposed that continually changed velocity profile should be adopted with the consideration of severe wear conditions, like running, jogging and jumping, etc.

\subsection{Contact pressure}

Contact pressure is another factor, which affects the accuracy of pre-clinical testing. Sonntag et al. [66] once reported that loading profiles of normal walking were 
Table 2 Various sliding velocity in the pre-clinical testing of artificial joints

\begin{tabular}{lll}
\hline Authors & Materials & Sliding velocity/(mm·s $^{-1}$ ) \\
\hline Saikko [46] & CoCr-UHMWPE & $0-31.4$ \\
Manhabosco et al. [52] & Alumina-Ti6Al4V & 32 \\
Gispert et al. [53] & Alumina/metal-UHMWPE & 46 \\
Wilches et al. [15] & Metal-UHMEPE & 58 \\
Zhang et al. [51] & Steel-PEEK & $200-1400$ \\
Kahyaoglu and Unal [54] & Stainless steel-UHMWPE & 500 \\
& & 1000 \\
\hline
\end{tabular}

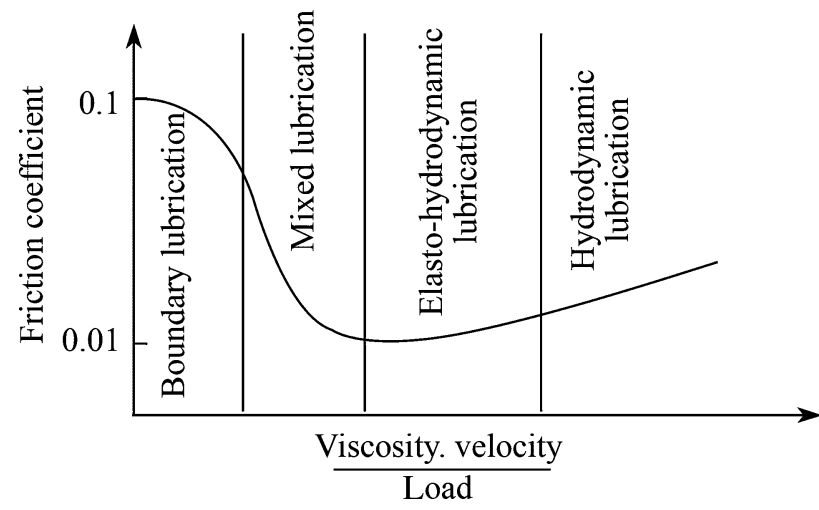

Fig. 4 Typical Stribeck curve describing the relationship between the friction coefficient and velocity [59]

different from that of climbing stairs or descending stairs, as a result, finding that the clinical outcome was not consistent with in vitro tests based on the normal walking data. Thus, they suggested that more severe loading conditions should be included in the testing profiles to get more precise pre-clinical testing data.

Mazzucco and Spector [67] investigated the relationship between contact pressure and wear rate of UHMWPE and claimed that increased contact pressure would result in more asperity interactions, thus increasing the wear rate. They also found that delamination would happen if the contact pressure of the implant-implant interface was higher than $7 \mathrm{MPa}$. Xiong [68] did a comprehensive research on the effect of load on the tribological performance of polymer-based artificial joints, finding that friction coefficient would decrease with the increased loading, while wear volume showed the opposite trend (see Fig. 5). Saikko [69] did a similar experiment and found that wear factor would decrease with the increased loading. In addition, it was put forward that the contact pressure during the in vitro testing should not exceed $2 \mathrm{MPa}$ and that larger contact stress might cause many protuberances on the UHMWPE surface, which was not consistent with the clinical situation. The same phenomenon was also

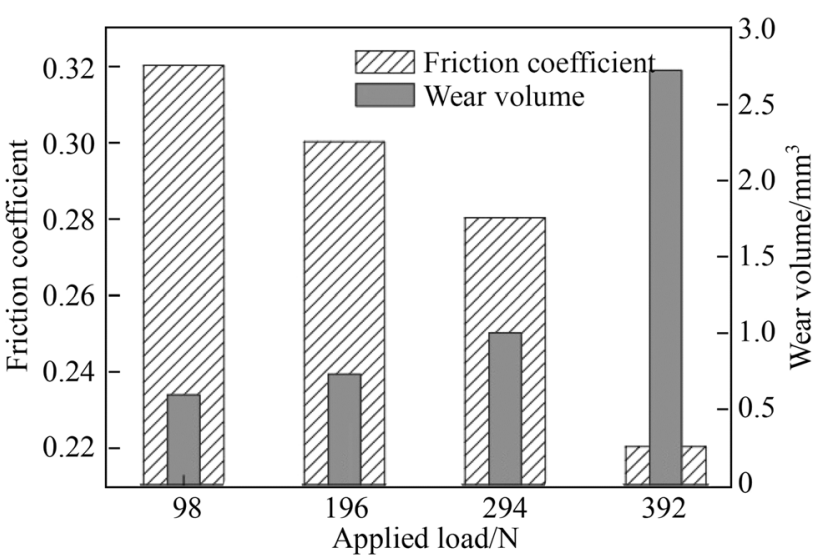

Fig. 5 Relationship between applied load and tribological performance of artificial joints [68]

observed by Wang et al. [70], and they said that such testing result could imply the partial contact of asperities for polymer-based bioimplants. It is widely recognised that metal-on-polymer configuration would always experience boundary lubrication regime, which implies that a thinner lubricant film would form when suffering higher contact pressure (see Fig. 4). In this situation, the friction coefficient would become larger, which was consistent with the laboratory test $[67,68]$. Meanwhile, when the contact stress gets larger, the increased number of asperities would result in the growth of wear rate (defined as volumetric loss/ million cycles). However, too large contact pressure would contribute to high frictional heating, which would soften the bearing surfaces. As a result, the polymer would get toughened, reducing the wear rate. Thus, it can be concluded that the wear rate of UHMWPE would firstly grow with the increasing contact pressure until a critical value, after which the wear rate would get reduced with the increase of contact pressure. Such critical value was once reported to be ranging from $2 \mathrm{MPa}$ to $3.5 \mathrm{MPa}$ [69]. However, it is proposed here that more strict protocols should be adopted to find this critical value in the future. In 
addition, there is a common sense for the polymer-based artificial joints that the wear rate of UHMWPE is not linearly proportional to the contact pressure, always with exponent value below 1 . In this case, the wear factor would decrease with the increase of contact pressure, which is consistent with the laboratory test.

As for hard-on-hard combination, there are a few investigations to find the correlation between tribological performance and the loading conditions. Myant et al. [71] found that the lubricant film thickness was very sensitive to the contact stress, and the film layer would get reduced with the increase of contact pressure. Leslie et al. [72] also reported that higher contact stress would result in a larger wear rate during the bedding-in period, while the steady wear rates were almost the same under different contact stresses. Generally, there is no comprehensive research to find the relationship between contact stress and wear resistance or friction force for hard-on-hard material combinations, and this gap should be filled in the future.

As we can see from Table 3, the contact pressure varies a lot among different scholars, ranging from $0.177 \mathrm{MPa}$ to $700 \mathrm{MPa}[9,15,73-78]$. Most researchers choose to use the constant loading to carry out the performance evaluation, which is easier to achieve compared to the changeable loading profile. However, the constant loading condition cannot replicate the real environment encountered by the implanted joints. Besides, it was reported that the edge contact in artificial hip joints could produce the most severe stress concentration, which could be up to $8 \mathrm{GPa}$ [79]. Such high stress could result in a high temperature between the contact surfaces, and change the surface topography. The difficulty in modelling hinders the understanding of wear mechanisms under extreme loading conditions, and the only data come from the explanted joints. As a result, there is a huge need to simulate this extremely severe loading condition in order to evaluate artificial joints thoroughly. Besides, it is suggested here, for all material combinations, that more evaluation indexes, including the morphology of wear particles, the topography of bearing surfaces and subsurface damage, should be checked in the future to establish a more thorough understanding for the effect of contact stress on the tribological performance. The surface topography and wear particles could be used to evaluate the wear mechanisms, while the subsurface damage is an important factor affecting the long-term performance of implanted joints.

\subsection{Hard particles}

It is recognised that hard particles, like bone cements or wear debris, would contribute to the change in the surface intergrity [80]. Fisher et al. [81] once reported that some scratches on the bearing surfaces, which were induced by the impingement of hard particles, would cause the increase of wear rate to a great extent. Hard particles were also found embedded inside the polymeric component of the explanted joints [82], and surface integrity of metallic parts was severely deteriorated (see Fig. 6) [83, 84]. Thus, the lifespan of artificial joints would get reduced greatly if some particles would enter the bearing interface. However, the adverse effect of hard particles on the tribological performance of artificial joints has not been considered in most laboratory tests, and this could partially explain the fact that the lifespan of implanted artificial joints is quite shorter than the predicted ones.

Cooper et al. [85] concluded that hard particles could increase the wear rate of artificial joints through two mechanisms. The first is that hard particles would enter the bearing interfaces and plough the contact surfaces directly. In this case, the surface integrity would get reduced and large quantities of wear particles could be produced. The other one is that the surface imperfections caused by the impingement and plow of hard particles would then result in much more material removal than the first mechanism. In addition, both clinical studies and laboratory testing have confirmed that these particles are mostly made up of

Table 3 Various contact pressure among different in vitro tests

\begin{tabular}{lll}
\hline Author & Materials & Contact pressure/MPa \\
\hline Wilches et al. [15] & Stainless steel-UHMWPE & $0.177,0.531,1.060,1.767$ \\
& Ti6Al4V-UHMWPE & $0.57-1.13$ \\
Chyr et al. [73] & CoCrMo-UHMWPE & 3.5 \\
Zhang et al. [74] & CoCrMo-CoCrMo & 16 \\
Choudhury et al. [9] & CoCrMo-optical glass & 51 \\
Guezmil et al. [75] & Stainless steel-UHMWPE & 112 \\
Yan el al. [76] & CoCrMo-CoCrMo & 560 \\
Sedlaček et al. [77] & Steel-steel & 700 \\
Kovalchenko et al. [78] & Steel-steel & \\
\hline
\end{tabular}



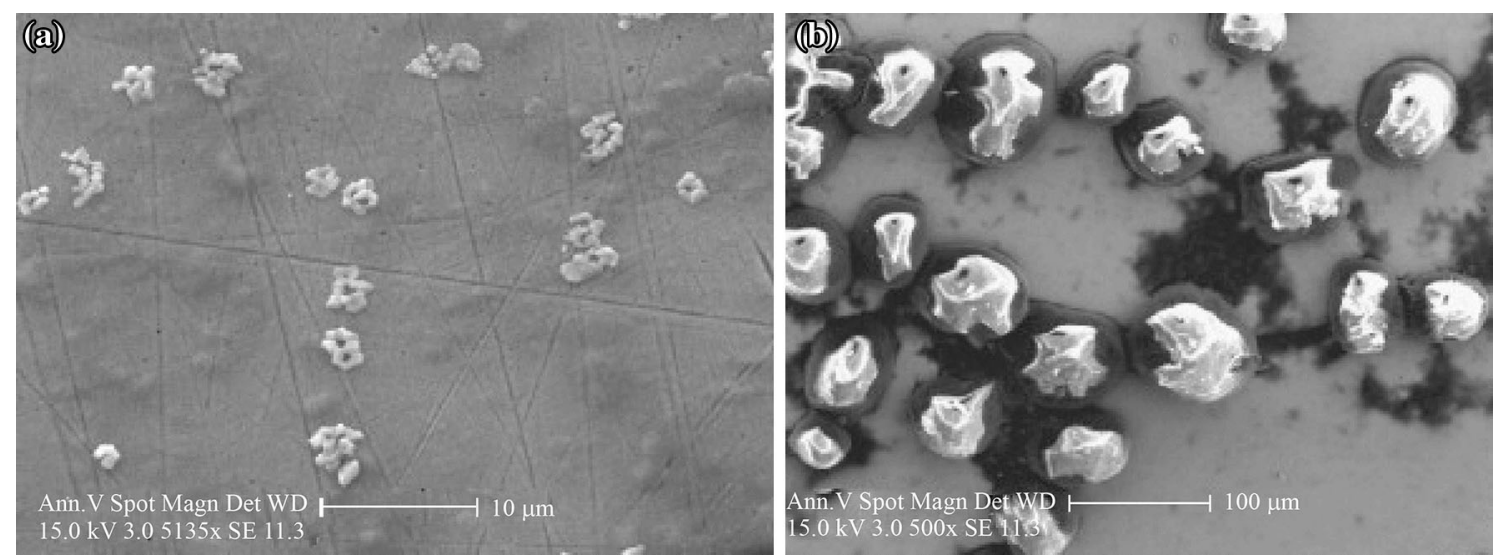

Fig. 6 Embedment of hard particles into the metallic bearing surfaces [84]

cement debris along with radiopaque additives, like barium sulphate and zirconia [86, 87]. Until now, two ways have been adopted by many researchers to test the influence of hard particles on the longevity of bio-implants. The first method is to manually make some scratches or small pits on the bearing surfaces. Dowson et al. [80] reported that even a single surface imperfection, like a scratch or indentation, could cause a significant increase in the wear rate of artificial joints. However, these artificial damages could not replicate the real clinical situation, which could be partially explained by the difficulty of producing a damaged surface geometry similar to that of the implanted ones. Besides, there would also be no particles embedded in the bearing surfaces since no hard debris was added into the testing lubricant, which was not consistent with the clinical situation [82]. As a result, it is optimal to test the performance of artificial joints with the existence of hard particles. Caravia et al. [88] put forward that the enrolment of bone cement in the testing lubricant could severely damage the metallic surfaces. They also found that the entering of particles into the bearing surfaces was mainly decided by the surface roughness, particle size and shape, and contact pressure. Besides, bone cement incorporated with polymerized zirconia was found to result in the largest amounts of wear loss. However, there are some problems with Caravia's experiment. It was reported that the sedimentation of particles could influence the testing result [89]. Nowadays, two methods are proposed to solve the precipitation of hard particles. The first one is to fix the particles onto the bearing surfaces [90], which could eliminate the sedimentation phenomenon. However, it was believed that some particles would move through the contact area and become free particles again [88]. In this case, the second method seems an optimal solution: particles-contaminated lubricants could be used to check the functionality of hard debris $[84,91]$. Particle size is another parameter which could affect the testing accuracy. It has been proved that large particles prefer to embed into the bearing surfaces while small debris is more likely to move through the contact area [88]. The clinical data on the particle size were rarely reported. Cooper et al. [85] revealed that the size of cement particles could range from several microns to $1 \mathrm{~mm}$. The explanted implants showed that the size of embedded particles varied between $20 \mu \mathrm{m}$ and $500 \mu \mathrm{m}$ [92]. The particle sizes used in Ref. [90] were from $100 \mu \mathrm{m}$ to $200 \mu \mathrm{m}$, while Caravia et al. [88] chosed to use a larger scale of hard particles, ranging from $5 \mu \mathrm{m}$ to $355 \mu \mathrm{m}$. Besides, the discrepancies in the particles' composite also made it hard to compare testing results from different research groups [84, 93].

It is proposed here that the focus of the research, related to the functionality of the hard particles, should be establishing a protocol, which stipulates the size range, composite and geometry of the particles used in the laboratory testing. Besides, the testing of newly-designed joints including the latest material combinations, surface structures, and coating layers, should be evaluated under the particles-contaminated environment.

\subsection{Lubricant}

Lubricant is a very important factor for the in vitro simulation test, and it could affect the testing results in many ways. The lubricant itself could cool down the temperature of the experiment environment. Tetreault and Kennedy [94] revealed that dry tests would produce a different wear mechanism compared to that under wet simulation. They explained that high temperature would exist between contact surfaces if no lubricant was used, and this could result in the delamination and material transfer. In addition, it is widely recognised that the proteins in the synovial fluids are very sensitive to the nanostructure on bearing surfaces, and the attached protein layers would affect the tribological performance of bio-implants. Lastly, the hydrodynamic 
pressure, which could separate the two bearing surfaces in vivo, would not exist if no lubricant was used. As a result, the functionality of some new designs, like surface texture, could not be fully evaluated without the existence of lubricant. However, it is difficult to gain sufficient volumes of natural synovial fluids [95], thus we need to use the simulated lubricants for laboratory tests. In general, there are three kinds of lubricants that have already been used for testing artificial joints: deionized water, saline solution and serum.

Deionized water was largely used as the testing lubricant in the last century due to a lack of the knowledge about in vivo wear mechanisms. After several decades of usage, many researchers have reported that the wear mechanisms of artificial joints under deionized water are significantly different from those of the in vivo situation. Copper et al. [96] confirmed that the soft polymer material was likely to transfer onto the hard surface under water-lubricated environment, and also found that it was rare to see the polymer transfer when using the protein-containing lubricant. Saikko [69] also put forward a similar concept that the absence of material transfer was a symbol of the active protein layer. Besides, the transfer of material could roughen the counterface (see Fig. 7, 97), resulting in the increase of wear rate. Wang et al. [95] found that the cross length of wear debris produced under the water lubricant was $2-3 \mathrm{~mm}$, which was too large compared to the clinical situation. At the same time, they claimed that bovine serum produced submicron particles, bearing more resemblance to the clinical findings. In this case, the protein-containing saline solution was used to evaluate the artificial joints [71]. There are two advantages in using such lubricant including the elimination of material transfer and the evaluation of the effect of specific proteins on the tribological performance of artificial joints. Gispert et al. [56] compared the functionality of hyaluronic acid (HA) and albumin based on the Hanks' balanced salt solution and

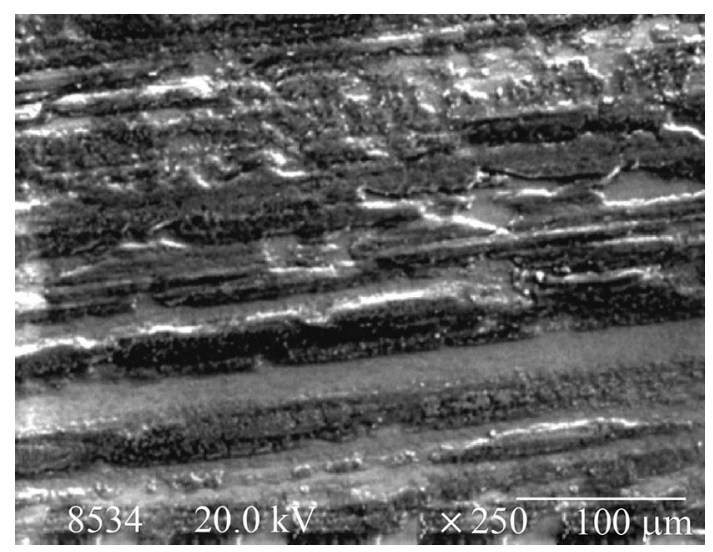

Fig. 7 SEM image of transferred UHMWPE layers on the metallic surface [97] concluded that the increasing volume of HA could result in a higher viscosity, while albumin protein could adsorb onto the bearing surfaces and improve the boundary lubricant. They also found that the existence of protein in the testing lubricant could inhibit the polymer transfer to the metallic surfaces. However, the saline solution could not fully test the lubrication properties of artificial joints since only several kinds of stuffs are incorporated into the fluid, which is different from the synovial fluids.

Serum is another popularly used lubricant for the in vitro testing, and it has already been regarded as the gold standard for laboratory evaluation. The viscosities and protein contents of commercial serums are different from each producer. The viscosity of serum solution could be altered by adding some lubricants, like carboxymethyl cellulose (CMC) fluids [65]. Thus, surem lubricants are suitable for drawing the Stribeck figures for different joints' designs to test the lubricant regimes. The protein concentration for the commercial serum lubricant is usually larger than $60 \mathrm{~g} / \mathrm{L}$, which is too large for laboratory tests, and this value could be customized by adding distilled water. Based on the ISO standard, the protein concentration for the in vitro evaluation should be around $20 \mathrm{~g} / \mathrm{L}$. However, it was reported that the lubricant around a healthy joint contains $18 \mathrm{~g} / \mathrm{L}$ of protein, while the values for the diseased joints range from $26 \mathrm{~g} / \mathrm{L}$ to $45 \mathrm{~g} / \mathrm{L}$ [98]. Thus, the protein concentrations are different among people, which requires the newly-designed joints to be tested under various protein concentrations. Besides, it is widely recognised that proteins would adsorb and form a boundary layer on the bearing surfaces [65]. As a result, wear mechanisms would get changed due to the precipitation of proteins. In addition, the activities of proteins are very sensitive to the surface structure, which means new designs, like surface roughness or textures, could have significantly different tribological performances under various protein contents. Also, different protein contents were believed to affect the laboratory testing results to a different extent [62]. Wang et al. [99] found that the ratio of albumin to $\gamma$-globulin could affect the tribological performance of artificial joints and claimed that the optimal value should be from 1 to 1.5 .

However, until now, most tests are conducted under the constant protein situation. Thus, it is suggested that various protein contents and concentrations should be used for preclinical evaluation of artificial joints, especially those with nanostructures. Besides, the visco-supplementation of the body fluids is a new area for improving the tribological performance of implanted joints [100]. Thus, the understanding of protein working mechanisms could further help increase the clinical service life. 


\section{Process evaluation}

The bio-reaction between wear debris and surrounding bones is considered as main failure mechanism for artificial joints. As a result, the main purpose of in vitro testing is to check the tribological property of bio-implants. Generally, it could be separated into three parts including the in vitro analysis of wear resistance, measurement of friction force and investigation of lubricant regime [101]. There are two methods to characterise the wear properties: the wear loss and changes in surface topography. The wear loss can be measured by the gravimetric or volumetric method. The surface topography will be inspected by different microscopes, including optical microscope, scanning electron microscope (SEM), and coordinate measuring machine (CMM). The optical method has been used recently to check the lubricant regime, and this will also be covered in this chapter. The friction force is detected based on the dynamometer installed in the machine.

\subsection{Gravimetric and volumetric method}

Two indexes are used to describe the testing results of in vitro wear tests, namely wear rate and wear factor. Wear rate is usually expressed in volumetric loss per million cycles $\left(\mathrm{mm}^{3} / 10^{6}\right.$ cycles), while wear factor considers the loading and distance profile, and it is usually written in the form of volumetric loss per newton and meter $\left(\mathrm{mm}^{3} /\right.$ $(\mathrm{N} \cdot \mathrm{m})$ ). Thus, both characterisation methods need the volume of wear loss. The gravimetric method is the most used method for measuring the wear loss by far.

A high accurate balance, with the resolution of $0.1 \mathrm{~g}$ or smaller, is used in the in vitro testing. Before using the balance, the sample should be cleaned and dried to eliminate the influence of fluid uptake and surface pollution $[102,103]$. Then, the volumetric value could be obtained through dividing the gravimetric value by the corresponding density. The main challenge for this method is to retain a pure sample without any contamination. Embedded particles or protein layers could be removed thoroughly. Reference experiment is considered as one way to reduce the effect of fluid uptake, which is achieved by soaking one sample in the testing fluid without applying loading and motion profile to it. However, it is widely accepted that the fluid uptake is different among testing samples, especially when the testing period is very short. Besides, the effects of loading on the uptaking rate has not been fully understood $[104,105]$. It could be said that reference experiment is suitable for long-term testing. For short evaluation process, drying procedure could solve the problem caused by fluid uptake.
The reason why researchers are more interested in volumetric loss other than the mass value is that the volumetric value could better reflect the change in surface topography, and it is approved by many scientists that even little changes to bearing surface could significantly affect the wear performance [80]. However, the density of testing material is not always uniformly distributed, which would affect the calculation accuracy. Besides, the gravimetric method could not cover the volumetric changes due to the creep and plastic deformation. As a result, the volumetric method seems a good solution. Here, the wear loss is directly measured by CMM in the form of volume. Thus, it could eliminate the effect of fluid uptake, creep, and plastic deformation.

\subsection{Optical method}

The thickness of lubricant film is an important factor for identifying the tribological performance of artificial joints. The thicker lubricant film reveals that there would be fewer interactions of surface asperities, resulting in smaller wear loss and friction coefficient. As a result, the way to predict or detect the thickness of lubricant film attracts many interests.

The prediction of lubricant regime is based on the corresponding Stribeck curve, which needs several testing data under various Sommerfeld parameters [56]. As a result, it needs several other comparative experiments to check the lubricant regime of a specific situation. In this case, optical method is used by many researchers since it can directly measure the thickness of lubricant film. Fan et al. [106] firstly adopted the optical method, using the CCD camera to capture the in situ image of the contact areas, to check the functionality of various fluid models. Nowadays, a typical setup for measuring the fluid thickness could be seen in Fig. 8, [101]. The pendulum configuration could provide a loading and motion profile similar to that of the clinical situation. The material combination is metal-onglass, which could help the camera receive interference lights. In this case, the image of chromatic interferogram could be obtained. However, for this method, a transparent material must be used during the measuring, which is contrary to the clinical situation. Thus, some improvements need to be made to detect the film thickness for other material combinations. It has been proved that optical method, incorporating the principle of fluorescence, is suitable for measuring the film thickness of opaque material combinations $[48,107]$. 


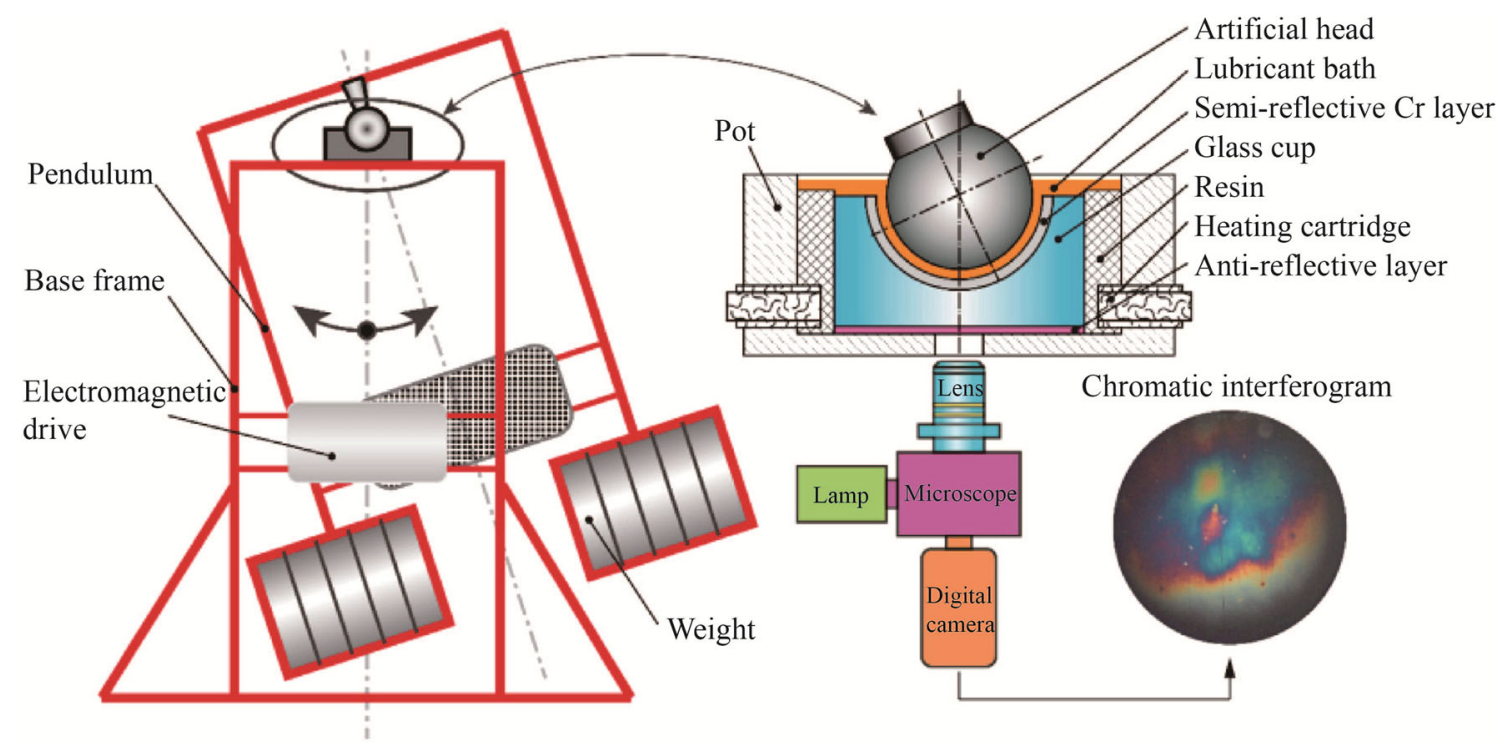

Fig. 8 Scheme of optical measurement [101]

\section{Conclusions}

Total joint replacement has seen great changes in the past decades, and the main advance has taken place in the improvement of wear resistance. Simultaneously, the methods to evaluate those newly-designed joints have advanced and could provide a better testing environment in which the wear mechanisms and lubricant regimes encountered by testing samples are similar to the implanted ones. The advances can be separated into three categories: testing approaches, evaluation parameters, and process evaluation.

The wear tracks provided by the pin/ball-on-disk have envolved from the undirectional sliding to the multidirectional motion. The latter motion profiles are believed to provide a better testing environment, and the aspect ratio will be the future research focus. Generally, the pin/ballon-disk testing is an easy and fast way to conduct the evaluation procedure, but the testing results might not represent the clinical situation. On the contrary, full joint simulator considers most testing indexes: geometry, anatomical position, motion and loading profiles. Thus, joint simulator could provide more accurate data compared to that based on the pin/ball-on-disk machine. Besides, five million cycles of testing must be conducted using the full joint simulator before the clinical application of any newlydesigned joints.

The testing paremeters of most previous laboratory evaluation aimed at simulating the normal walking condition, which is believed to be the main motion situation of the daily exercise. Such testing environment is recognised to produce a lower wear rate than the clinical situation, and more efforts have been made to achieve severe wear condition. Four main parameters: sliding velocity, contact pressure, hard particles and lubricant, are important in achieving the desired severe situation. In the future, the research focus should be in understanding the functionality of hard particles and lubricant. For the hard particles, the main research gap is the lack in the protocal, stipulating the size, shape and composite of hard debris. The effects of specific lubricant contents, especially the proteins, on the tribological performance of artificial joints should also be investigated in the future.

Wear is believed to be the main failure mechanism of bio-implants. As a result, wear loss is widely measured by researchers. The detection method could be gravimetric or volumetric analysis. The main problem in the gravimetric method is that it is difficult to eliminate the effect of fluid uptake, especially when the testing period is short. Besides, the changes in the surface topography due to creep or plastic deformation could not be measured by the gravimetric method. Thus, it is suggested here that gravimetric method could still be the main measuring procedure, while volumetric analysis should be the auxiliary tool for the in vitro evaluation.

Acknowledgements The authors would like to thank R. Melentiev and C. Kang for their valuable discussions. Acknowledgments are extended to the support from the Science Foundation Ireland (Grant Nos. 15/RP/B3208 and 16RC3872) and the National Natural Science Foundation of China (Grant Nos. 51320105009 and 61635008).

Open Access This article is distributed under the terms of the Creative Commons Attribution 4.0 International License (http://crea tivecommons.org/licenses/by/4.0/), which permits unrestricted use, distribution, and reproduction in any medium, provided you give appropriate credit to the original author(s) and the source, provide a link to the Creative Commons license, and indicate if changes were made. 


\section{References}

1. Kang CW, Fang FZ (2018) State of the art of bioimplants manufacturing: part I. Adv Manuf 6(1):20-40

2. Kang CW, Fang FZ (2018) State of the art of bioimplants manufacturing: part II. Adv Manuf 6(2):137-154

3. Kurtz S, Ong K, Lau E et al (2007) Projections of primary and revision hip and knee arthroplasty in the United States from 2005 to 2030. J Bone Joint Surg Am 89(4):780-785

4. Rahaman MN, Yao A, Bal BS et al (2007) Ceramics for prosthetic hip and knee joint replacement. J Am Ceram Soc 90(7):1965-1988

5. Ching HA, Choudhury D, Nine MJ et al (2014) Effects of surface coating on reducing friction and wear of orthopaedic implants. Sci Technol Adv Mater 15(1):014402

6. Fang FZ (2016) Atomic and close-to-atomic scale manufacturing - a trend in manufacturing development. Front Mech Eng 11(4):325-327

7. Fang FZ (2014) Manufacturing III (Invited lecture). University College Dublin, Dublin

8. Yamane S, Moro T, Kyomoto M et al (2017) Wear resistance of vitamin E-blended polyethylene tibial insert by MPC polymer grafting. Bone Joint J 99(s 6):104

9. Choudhury D, Rebenda D, Sasaki S et al (2018) Enhanced lubricant film formation through micro-dimpled hard-on-hard artificial hip joint: An in-situ observation of dimple shape effects. J Mech Behav Biomed Mater 81:120-129

10. Koseki H, Tomita M, Yonekura A et al (2017) Effect of carbon ion implantation on the tribology of metal-on-metal bearings for artificial joints. Int J Nanomed 12:4111-4116

11. Chen Y, Li Y, Kurosu S et al (2015) Analysis of run-in-stage wear behavior and contact mechanics of metal-on-metal hip joint bearings with different radial clearances. Mater Trans 56(6):826-834

12. Affatato S (2012) Wear of orthopaedic implants and artificial joints. Elsevier, Amsterdam

13. Bowsher J, Shelton J (2001) A hip simulator study of the influence of patient activity level on the wear of crosslinked polyethylene under smooth and roughened femoral conditions. Wear 250(1-12):167-179

14. Chen Y, Li Y, Koizumi Y et al (2017) Effects of carbon addition on wear mechanisms of CoCrMo metal-on-metal hip joint bearings. Mater Sci Eng C 76:997-1004

15. Wilches L, Uribe J, Toro A (2008) Wear of materials used for artificial joints in total hip replacements. Wear 265(1-2):143-149

16. Ghosh S, Choudhury D, Das NS, et al (2014) Tribological role of synovial fluid compositions on artificial joints-a systematic review of the last 10 years. Lubr Sci 26(6):387-410

17. Fisher J, Dowson D, Hamdzah H et al (1994) The effect of sliding velocity on the friction and wear of UHMWPE for use in total artificial joints. Wear 175(1-2):219-225

18. Bowsher J, Shelton J (2000) The influence of stumbling on the wear of ultra-high molecular weight polyethylene. In: Proceedings of the 6th world biomaterials congress transactions, 2000, p 867

19. Bigsby R, Hardaker C, Fisher J (1997) Wear of ultra-high molecular weight polyethylene acetabular cups in a physiological hip joint simulator in the anatomical position using bovine serum as a lubricant. Proc Inst Mech Eng Part H J Eng Med 211(3):265-269

20. Kurtz SM (2009) UHMWPE biomaterials handbook: Ultra high molecular weight polyethylene in total joint replacement and medical devices. Academic Press, London
21. Baykal D, Siskey R, Haider H et al (2014) Advances in tribological testing of artificial joint biomaterials using multidirectional pin-on-disk testers. $\mathrm{J}$ Mech Behav Biomed Mater 31:117-134

22. McKellop H, Clarke I, Markolf K et al (1978) Wear characteristics of UHMW polyethylene: A method for accurately measuring extremely low wear rates. J Biomed Mater Res Part A 12(6):895-927

23. Shen G, Fang FZ, Kang CW (2018) Tribological performance of bioimplants: A comprehensive review. Nanotechnol Precis Eng 1(2):107-122

24. Archard J (1953) Contact and rubbing of flat surfaces. J Appl Phys 24(8):981-988

25. Wang A (2001) A unified theory of wear for ultra-high molecular weight polyethylene in multi-directional sliding. Wear 248(1-2):38-47

26. Wang A, Polineni V, Essner A et al (1997) The significance of nonlinear motion in the wear screening of orthopaedic implant materials. J Test Eval 25(2):239-245

27. Saikko V, Ahlroos T (1999) Type of motion and lubricant in wear simulation of polyethylene acetabular cup. Proc Inst Mech Eng Part H J Eng Med 213(4):301-310

28. Pooley CM, Tabor D (1972) Friction and molecular structure: The behaviour of some thermoplastics. Proc R Soc Lond A 329(1578):251-274

29. Bragdon CR, O'Connor DO, Lowenstein JD et al (2001) A new pin-on-disk wear testing method for simulating wear of polyethylene on cobalt-chrome alloy in total hip arthroplasty. J Arthroplasty 16(5):658-665

30. Turell M, Friedlaender G, Wang A et al (2005) The effect of counterface roughness on the wear of UHMWPE for rectangular wear paths. Wear 259(7-12):984-991

31. Turell M, Wang A, Bellare A (2003) Quantification of the effect of cross-path motion on the wear rate of ultra-high molecular weight polyethylene. Wear 255(7-12):1034-1039

32. Bennett D, Humphreys L, O'brien S et al (2008) The influence of wear paths produced by hip replacement patients during normal walking on wear rates. J Orthop Res 26(9):1210-1217

33. Davey S, Orr J, Buchanan F et al (2004) Measurement of molecular orientation in retrieved ultra-high-molecular-weight polyethylene (UHMWPE) hip sockets using Fourier-transform infrared spectroscopy. Strain 40(4):203-210

34. Saikko V, Calonius O, Keränen J (2004) Effect of slide track shape on the wear of ultra-high molecular weight polyethylene in a pin-on-disk wear simulation of total hip prosthesis. J Biomed Mater Res B Appl Biomater 69(2):141-148

35. Korduba L, Wang A (2011) The effect of cross-shear on the wear of virgin and highly-crosslinked polyethylene. Wear 271(9-10):1220-1223

36. Firkins P, Tipper J, Saadatzadeh M et al (2001) Quantitative analysis of wear and wear debris from metal-on-metal hip prostheses tested in a physiological hip joint simulator. Bio-Med Mater Eng 11(2):143-157

37. Tipper J, Firkins P, Besong A et al (2001) Characterisation of wear debris from UHMWPE on zirconia ceramic, metal-onmetal and alumina ceramic-on-ceramic hip prostheses generated in a physiological anatomical hip joint simulator. Wear 250(1-12):120-128

38. Ali M, Al-Hajjar M, Partridge S et al (2016) Influence of hip joint simulator design and mechanics on the wear and creep of metal-on-polyethylene bearings. Proc Inst Mech Eng Part H J Eng Med 230(5):389-397

39. Moro T, Takatori Y, Kyomoto M et al (2014) Long-term hip simulator testing of the artificial hip joint bearing surface grafted with biocompatible phospholipid polymer. J Orthop Res 32(3):369-376 
40. Herrmann S, Kaehler M, Grawe R et al (2015) Physiologicallike testing of the dislocation stability of artificial hip joints. In: Wenger P, Flores P (eds) New trends in mechanism and machine science. Springer, Berlin, pp 659-667

41. Kawanabe K, Clarke IC, Tamura J et al (2001) Effects of A-P translation and rotation on the wear of UHMWPE in a total knee joint simulator. J Biomed Mater Res Part A 54(3):400-406

42. Dowson D, Gillis B, Atkinson J (1985) Penetration of metallic femoral components into polymeric tibial components observed in a knee joint simulator. ACS Publications, Washington

43. Tipper J, Galvin A, Williams S et al (2006) Isolation and characterization of UHMWPE wear particles down to ten nanometers in size from in vitro hip and knee joint simulators. J Biomed Mater Res Part A 78(3):473-480

44. McKellop HA, D'lima D (2008) How have wear testing and joint simulator studies helped to discriminate among materials and designs? JAAOS J Am Acad Orthop Surg 16:S111-S119

45. Affatato S, Ruggiero A, Merola M (2015) Advanced biomaterials in hip joint arthroplasty. A review on polymer and ceramics composites as alternative bearings. Composites Part B Engineering 83:276-283

46. Saikko V (2017) Effect of contact area on the wear of ultrahigh molecular weight polyethylene in noncyclic pin-on-disk tests. Tribol Int 114:84-87

47. Zhang T, Deng Q, Liu B et al (2015) Wear and corrosion properties of diamond like carbon (DLC) coating on stainless steel, CoCrMo and Ti6Al4V substrates. Surf Coat Technol 273:12-19

48. Nečas D, Vrbka M, Urban F et al (2016) The effect of lubricant constituents on lubrication mechanisms in hip joint replacements. J Mech Behav Biomed Mater 55:295-307

49. Deng Y, Xiong D (2015) Fabrication and properties of UHMWPE grafted with acrylamide polymer brushes. J Polym Res 22(10): 195

50. Krismer M (2017) Sports activities after total hip arthroplasty. EFORT Open Rev 2(5):189-194

51. Zhang G, Zhang C, Nardin P et al (2008) Effects of sliding velocity and applied load on the tribological mechanism of amorphous poly-ether-ether-ketone (PEEK). Tribol Int 41(2):79-86

52. Manhabosco TM, Barboza APM, Batista RJC et al (2013) Corrosion, wear and wear-corrosion behavior of graphite-like $a$ $\mathrm{C}$ : $\mathrm{H}$ films deposited on bare and nitrided titanium alloy. Diam Relat Mater 31:58-64

53. Gispert M, Serro A, Colaco R et al (2006) Friction and wear mechanisms in hip prosthesis: comparison of joint materials behaviour in several lubricants. Wear 260(1-2):149-158

54. Kahyaoglu O, Unal H (2012) Friction and wear behaviours of medical grade UHMWPE at dry and lubricated conditions. Int $\mathrm{J}$ Phys Sci 7(16):2478-2485

55. Dressler MR, Strickland MA, Taylor M et al (2011) Predicting wear of UHMWPE: decreasing wear rate following a change in direction. Wear 271(11-12):2879-2883

56. Welghtman B, Light D (1986) The effect of the surface finish of alumina and stainless steel on the wear rate of UHMW polyethylene. Biomaterials 7(1):20-24

57. Streicher R, Weber H, Schoen R et al (1993) Wear resistant couplings for longer lasting articulating total joint replacements. Adv Biomater 10:179-186

58. Liew KW, Kok CK, Efzan ME (2016) Effect of EDM dimple geometry on friction reduction under boundary and mixed lubrication. Tribol Int 101:1-9

59. Dresel W (2007) Lubricants and lubrication. Wiley, New York

60. Houdková Š, Šperka P, Repka M et al (2017) Shifted laser surface texturing for bearings applications. J Phys Conf Ser 843(1):012076
61. Czichos H, Habig K (1992) Tribologie Handbuch: Systemanalyse, Prüftechnik, Werkstoffe und Konstruktionselemente. Vieweg Verlag, Wiesbaden

62. Nečas D, Sawae Y, Fujisawa T et al (2017) The influence of proteins and speed on friction and adsorption of metal/ UHMWPE contact pair. Biotribology 11:51-59

63. Saikko V (2003) Effect of lubricant protein concentration on the wear of ultra-high molecular weight polyethylene sliding against a CoCr counterface. J Tribol 125(3):638-642

64. Lu Z, McKellop H (1997) Frictional heating of bearing materials tested in a hip joint wear simulator. Proc Inst Mech Eng Part H J Eng Med 211(1):101-108

65. Scholes S, Unsworth A (2006) The effects of proteins on the friction and lubrication of artificial joints. Proc Inst Mech Eng Part H J Eng Med 220(6):687-693

66. Sonntag R, Reinders J, Rieger JS et al (2013) Hard-on-hard lubrication in the artificial hip under dynamic loading conditions. Plos One 8(8):e71622

67. Mazzucco D, Spector M (2003) Effects of contact area and stress on the volumetric wear of ultrahigh molecular weight polyethylene. Wear 254(5-6):514-522

68. Xiong DS (2005) Friction and wear properties of UHMWPE composites reinforced with carbon fiber. Mater Lett 59(2-3):175-179

69. Saikko V (2006) Effect of contact pressure on wear and friction of ultra-high molecular weight polyethylene in multidirectional sliding. Proc Inst Mech Eng Part H J Eng Med 220(7):723-731

70. Wang A, Essner A, Klein R (2001) Effect of contact stress on friction and wear of ultra-high molecular weight polyethylene in total hip replacement. Proc Inst Mech Eng Part H J Eng Med 215(2):133-139

71. Myant C, Underwood R, Fan J et al (2012) Lubrication of metalon-metal hip joints: The effect of protein content and load on film formation and wear. J Mech Behav Biomed Mater 6:30-40

72. Leslie I, Williams S, Brown C et al (2008) Effect of bearing size on the long-term wear, wear debris, and ion levels of large diameter metal-on-metal hip replacements-an in vitro study. J Biomed Mater Res B Appl Biomater 87(1):163-172

73. Chyr A, Qiu M, Speltz JW et al (2014) A patterned microtexture to reduce friction and increase longevity of prosthetic hip joints. Wear 315(1-2):51-57

74. Zhang H, Qin LG, Hua M et al (2015) A tribological study of the petaloid surface texturing for $\mathrm{Co}-\mathrm{Cr}-\mathrm{Mo}$ alloy artificial joints. Appl Surf Sci 332:557-564

75. Guezmil M, Bensalah W, Mezlini S (2016) Effect of bio-lubrication on the tribological behavior of UHMWPE against M30NW stainless steel. Tribol Int 94:550-559

76. Yan Y, Dowson D, Neville A (2013) In-situ electrochemical study of interaction of tribology and corrosion in artificial hip prosthesis simulators. J Mech Behav Biomed Mater 18:191-199

77. Sedlaček M, Podgornik B, Vižintin J (2012) Correlation between standard roughness parameters skewness and kurtosis and tribological behaviour of contact surfaces. Tribol Int 48:102-112

78. Kovalchenko A, Ajayi O, Erdemir A et al (2011) Friction and wear behavior of laser textured surface under lubricated initial point contact. Wear 271(9-10):1719-1725

79. Liu F, Fisher J (2017) Effect of an edge at cup rim on contact stress during micro-separation in ceramic-on-ceramic hip joints. Tribol Int 113:323-329

80. Dowson D, Taheri S, Wallbridge N (1987) The role of counterface imperfections in the wear of polyethylene. Wear 119(3):277-293

81. Fisher J, Firkins P, Reeves E et al (1995) The influence of scratches to metallic counterfaces on the wear of ultra-high 
molecular weight polyethylene. Proc Inst Mech Eng Part H J Eng Med 209(4):263-264

82. Dowling JM, Atkinson J, Dowson D et al (1978) The characteristics of acetabular cups worn in the human body. J Bone Joint Surg Br 60(3):375-382

83. Isaac G, Atkinson J, Dowson D et al (1986) The role of cement in the long term performance and premature failure of Charnley low friction arthroplasties. Eng Med 15(1):19-22

84. Kubo K, Clarke I, Sorimachi T et al (2009) Aggressive 3rd-body wear challenge to highly crosslinked polyethylene: A hip simulator model. Wear 267(5-8):734-742

85. Cooper J, Dowson D, Fisher J et al (1991) Ceramic bearing surfaces in total artificial joints: Resistance to third body wear damage from bone cement particles. J Med Eng Technol 15(2):63-67

86. Atkinson J, Dowson D, Isaac G et al (1985) Laboratory wear tests and clinical observations of the penetration of femoral heads into acetabular cups in total replacement hip joints: II: A microscopical study of the surfaces of Charnley polyethylene acetabular sockets. Wear 104(3):217-224

87. Atkinson J, Dowson D, Isaac J et al (1985) Laboratory wear tests and clinical observations of the penetration of femoral heads into acetabular cups in total replacement hip joints: III: The measurement of internal volume changes in explanted Charnley sockets after 2-16 years in vivo and the determination of wear factors. Wear 104(3):225-244

88. Caravia L, Dowson D, Fisher J et al (1990) The influence of bone and bone cement debris on counterface roughness in sliding wear tests of ultra-high molecular weight polyethylene on stainless steel. Proc Inst Mech Eng Part H J Eng Med 204(1):65-70

89. Zietz C, Fabry C, Reinders J et al (2015) Wear testing of total hip replacements under severe conditions. Expert Rev Med Devices 12(4):393-410

90. Hembus J, Lux L, Jackszis M et al (2018) Wear analysis of cross-linked polyethylene inserts articulating with alumina and ion-treated cobalt-chromium femoral heads under third-body conditions. Wear 402:216-223

91. Affatato S, Bersaglia G, Foltran I et al (2002) The performance of gamma- and EtO-sterilised UHWMPE acetabular cups tested under severe simulator conditions. Part 1: Role of the third-body wear process. Biomaterials 23(24):4839-4846

92. Lundberg HJ, Liu SS, Callaghan JJ et al (2007) Association of third body embedment with rim damage in retrieved acetabular liners. Clin Orthop Relat Res 465:133-139

93. Oberbach T, Begand S, Kaddick C (2009) Surface resistance of dispersion ceramics against third body abrasion. Key Eng Mater 396-398:161-164
94. Tetreault DM, Kennedy FE (1989) Friction and wear behavior of ultrahigh molecular weight polyethylene on $\mathrm{Co}-\mathrm{Cr}$ and titanium alloys in dry and lubricated environments. Wear 133(2):295-307

95. Wang A, Essner A, Stark C et al (1996) Comparison of the size and morphology of UHMWPE wear debris produced by a hip joint simulator under serum and water lubricated conditions. Biomaterials 17(9):865-871

96. Cooper J, Dowson D, Fisher J (1993) The effect of transfer film and surface roughness on the wear of lubricated ultra-high molecular weight polyethylene. Clinical Materials 14(4):295-302

97. Onate J, Comin M, Braceras I et al (2001) Wear reduction effect on ultra-high-molecular-weight polyethylene by application of hard coatings and ion implantation on cobalt chromium alloy, as measured in a knee wear simulation machine. Surf Coat Technol 142:1056-1062

98. Geigy D (1970) Scientific tables, 7th edn. Geigy Pharmaceuticals, Basel

99. Wang A, Essner A, Schmidig G (2004) The effects of lubricant composition on in vitro wear testing of polymeric acetabular components. J Biomed Mater Res Part B Appl Biomater 68(1):45-52

100. Dowson D (2012) Bio-tribology. Faraday Discuss 156(1):9-30

101. Nečas D, Vrbka M, Rebenda D et al (2018) In situ observation of lubricant film formation in THR considering real conformity: The effect of model synovial fluid composition. Tribol Int 117:206-216

102. Aurora A, DesJardins J, Joseph P et al (2006) Effect of lubricant composition on the fatigue properties of ultra-high molecular weight polyethylene for total knee replacement. Proc Inst Mech Eng Part H J Eng Med 220(4):541-551

103. Hu P, Liu R, Liu J et al (2014) Advanced Stellite alloys with improved metal-on-metal bearing for hip implants. Mater Des 60:424-432

104. Blunt L, Bills P, Jiang X et al (2009) The role of tribology and metrology in the latest development of bio-materials. Wear 266(3-4):424-431

105. Bills P, Brown L, Jiang X et al (2005) A metrology solution for the orthopaedic industry. J Phys Conf Ser 13:316-319

106. Fan J, Myant C, Underwood R et al (2011) Inlet protein aggregation: A new mechanism for lubricating film formation with model synovial fluids. Proc Inst Mech Eng Part H J Eng Med 225(7):696-709

107. Nečas D, Vrbka M, Křupka I et al (2016) Lubrication within hip replacements: implication for ceramic-on-hard bearing couples. J Mech Behav Biomed Mater 61:371-383 